\title{
Dirac gets the jitters
}

In 1928, British physicist Paul Dirac derived his famous equation for the relativistic quantum mechanics of spin-1/2 particles. Following his unique aesthetic sense for mathematical elegance, Dirac sought a linear differential equation with only a first-order derivative in time, akin to the Schrödinger equation, that would reflect the electron's intrinsic spin and also lead to the relativistic relationship between energy and momentum, $E^{2}=p^{2} c^{2}+m^{2} c^{4}$. Dirac found his equation; and much more.

Dirac's equation has an infinite number of solutions with negative energies. This obviously needs some explaining, as real electrons of positive energy exist stably in free space, and never seem to plunge into that abyss of negativity. Dirac supposed that an invisible sea of negative-energy electrons fills that abyss, preventing ordinary electrons from falling into it. Incredibly, this idea led him to predict the existence of the positron, discovered five years later.

Dirac's equation can no longer be considered mysterious, given its central position in modern physics. But 60 years later, some of the equation's features have yet to be explored experimentally. Modern semiconductor technologies could help to change that, bringing some of the strangest effects finally within experimental reach.

In 1930, Erwin Schrödinger showed that Dirac's equation implies an unexpected relativistic interaction between an electron's translational motion and spin, which should lead to a violent oscillation of the particle at very high frequencies and over distances of roughly one Compton wavelength. This phenomenon of zitterbewegung - from the German word for 'jitter' - has never been observed directly. Given its predicted frequency (about $10^{21} \mathrm{~Hz}$ ), it might be some time before a physicist, in the manner of botanist Robert Brown, peers through a microscope of some design to see the electron erratically bouncing around.

But, as several physicists have recently proposed, it might soon be possible to probe a close analogue of zitterbewegung by recreating the mathematics of Dirac's equation in a quantum wire (J. Schliemann et al. Phys. Rev. Lett. 94, 206801; 2005).

For Dirac's electrons, zitterbewegung takes place whenever the electron wavefunction includes both positive and negative energy components; this is generally the case, as it takes both sets of states to build

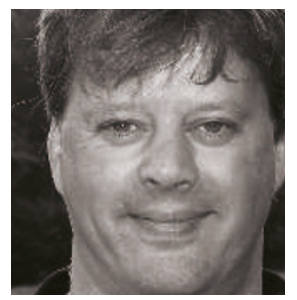

IT MIGHT BE POSSIBLE TO

PROBE A CLOSE

ANALOGUE OF ZITTERBEWEGUNG BY RECREATING THE

MATHEMATICS OF DIRAC'S EQUATION IN A QUANTUM WIRE.

\section{QUANTUM WIRE.}

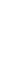



\section{Lost in Los Angeles}

I was driving around Los Angeles a few weeks ago, lost, and relying on a global positioning system receiver to guide me through the maze of freeways and unfamiliar streets. In the unending traffic jams, I had plenty of time to ponder and, surprisingly perhaps, my thoughts turned to Einstein.

2005 has been named World Year of Physics, to celebrate the centenary of Albert Einstein's 'miracle year', in which he wrote five seminal papers. The whole world is familiar with $E=m c^{2}$, but really it was Einstein's development of general relativity a decade later — and the dramatic confirmation in 1919 of the predicted bending of light, a consequence of interpreting gravity as a curvature of space - that catapulted him to the kind of superstar status that warrants a whole year being named in his honour.

General relativity was perhaps the most objectively cerebral theoretical development in modern physics. It was not a response to an experimental problem (the precession of the perihelion of Mercury didn't seem to need a revolution in physics to explain it), and direct laboratory confirmation only took place over a quarter century after the theory was first written down.

But without that exotic theory of spacetime, I wouldn't have been able to find my way around LA. Global positioning systems rely on the timing of signals sent from several satellites located thousands of miles apart. However, the internal clocks of GPS satellites tick at rates that are shifted, according to gen-

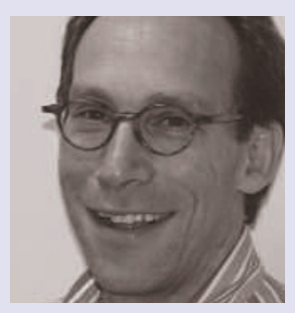

WITHOUT

EINSTEIN'S

EXOTIC THEORY

OF SPACETIME, I

WOULDN'T HAVE

BEEN ABLE TO

FIND MY WAY

AROUND LA. eral relativity, depending on their position in the Earth's gravitational field. Without correcting for that, GPS would not work properly.

Einstein didn't develop general relativity because he wanted to find a better way to track his own position, even if the technology had been available. But it is hard to think of a better example than this of the cross-germination between fundamental scientific investigation and technological innovation.

In this World Year of Physics, as economic pressures have led to cuts in funding for fundamental research in many industrialized countries, it is worth reflecting on this connection - at least the next time you're caught in traffic and looking for an alternative route. Lawrence M. Krauss 